\title{
DIVIMP Simulations of ${ }^{13} \mathrm{C}$ Puffing Experiments in ASDEX Upgrade L-mode Plasma
}

\author{
T. Makkonen*a, M. Groth ${ }^{\mathrm{a}}$, T. Kurki-Suonio ${ }^{\mathrm{a}}$, K. Krieger ${ }^{\mathrm{b}}$, L. Aho-Mantila $^{\mathrm{c}}$, A. Hakola $^{\mathrm{c}}$, \\ J. Likonen ${ }^{\mathrm{c}}$, H.W. Müller ${ }^{\mathrm{b}}$, and the ASDEX Upgrade Team \\ ${ }^{a}$ Aalto University School of Science and Technology, Association Euratom-Tekes, FI-00076 AALTO, Finland \\ ${ }^{b}$ Max-Planck-Institut fur Plasmaphysik, EURATOM Association, Boltzmannstrasse 2, 85748 Garching, Germany \\ ${ }^{c}$ VTT Technical Research Centre of Finland, Association Euratom-Tekes, 02044 VTT, Finland
}

\begin{abstract}
Carbon migration has been investigated both experimentally and numerically by injecting isotopically marked methane $\left({ }^{13} \mathrm{CH}_{4}\right)$ at trace levels into a series of identical low-density, low-confinement mode discharges at the last day of the 2007 ASDEX Upgrade experimental campaign. The experiment is simulated with the DIVIMP code to test which assumptions are needed to reproduce the observed deposition pattern. Including a realistic poloidal flow profile was observed to be crucial in replicating the experimental results.
\end{abstract}

JNM keywords: C0100, I0100, T0900

PSI-19 keywords: Carbon impurities, ASDEX-Upgrade, DIVIMP, OSM model, SOL plasma flow

PACS: 52.25.Fi, 52.25.Vy, 52.40.Hf, 52.40.Kh

*Corresponding author address: P.O.Box 14100 FI-00076 AALTO Finland

*Corresponding author E-mail: toni.makkonen@tkk.fi

Presenting author: Toni Makkonen

Presenting author E-mail: toni.makkonen@tkk.fi 


\section{Introduction}

Understanding impurity transport in the scrape off layer (SOL) plasma is crucial for predicting and controlling long term material erosion and redeposition patterns in a tokamak device. Co-deposition of hydrogen isotopes, including tritium, in carbon-rich surface layers and the subsequent build-up of a tritium inventory is of special concern for ITER.

To this end, isotopically marked tracer elements can be injected into tokamaks. Here we concentrate on an injection experiment performed at the the end of the 2007 ASDEX Upgrade [1] experimental campaign, where isotopically marked methane $\left({ }^{13} \mathrm{CH}_{4}\right)$ was injected into 11 identical lower single null L-mode discharges \#22573 - \#22585 [2]. The plasma parameters for the discharges were: toroidal magnetic field $B_{t}=-2.5 \mathrm{~T}$, plasma current $I_{p}=0.8 \mathrm{MA}$ with the gradient B drift pointing towards the active divertor, line averaged density $n_{e}=3.3 \times 10^{19} \mathrm{~m}^{-3}$, and external heating power $P_{\mathrm{aux}}=0.75 \mathrm{MW}$ achieved by electron cyclotron resonance heating. Methane was injected at the outer midplane port during the plasma flat-top phase. From there it penetrated into the SOL where it broke up and the carbon was released. As the valve is located approximately $1 \mathrm{~m}$ radially away from the plasma surface, the point source of the methane is spread over the port volume. Considering this large source with the large shear in the SOL, one would expect a fairly symmetric toroidal deposition [2]. The injection experiment was done at the end of the campaign in order to limit re-erosion. A total of $2.7 \times 10^{22}{ }^{13} \mathrm{C}$ atoms were injected. The subsequent deposition was measured using secondary ion mass spectrometry (SIMS) at VTT from a full poloidal set of lower divertor tiles and from selected main chamber tiles from the same toroidal location [2].

The 2007 carbon injection experiment is simulated with the DIVIMP code to test which assumptions are needed to reproduce the observed deposition pattern. Section 2 introduces the DIVIMP program and describes the plasma background generation. Section 3 presents the results, while a short summary is given in section 4 . 


\section{The DIVIMP Code and Background Generation}

The DIVIMP code $[3,4]$ combines a test particle Monte Carlo code for impurity ions and neutrals with various onion skin model (OSM) plasma background solvers and the neutral particle Monte Carlo code Eirene [5].

In this work, the particles were introduced as a point source at the outer midplane SOL and followed until they strike the divertor or exit the computational grid. The break-up chain of methane could, in principle, be modeled with DIVIMP, but was not done in this work. Instead, the source location was varied. Other scanned parameters were the spatially constant diffusion coefficient and the ion periphery option implemented in DIVIMP. The initial charge state was assumed +1 , and the initial energy of the ions was set to $0.3 \mathrm{eV}$ in accordance with the Franck-Cordon dissociation energy.

When exiting the computational grid, the particles are deposited on the closest wall element. The grid does not extend all the way to the walls in the upper parts of the machine, and possible computational effects of this limitation can be tested using the ion periphery option in DIVIMP. This option allows extending the computational area from the main SOL into the halo plasma of a specified width. In this extended area, the impurity ions can either diffuse through this specified width to the walls, or return back into the main SOL.

Only primary carbon deposition was studied. Erosion could play an important role in shaping the deposition profile. This work, however, mainly studies large scale deposition and relies on the assumption that erosion during the 11 last discharges does not critically affect the ratios of deposition between various parts of the machine.

The plasma background used in this work was generated with the OSM solver 22 [6] in DIVIMP coupled to the neutral code Eirene. The OSM solver uses measured temperature and density profiles at the target plates as input and computes the density, flow velocity, electron temperature, ion temperature, and parallel electric field upstream. The electron density and temperature data were taken from Langmuir probes embedded in the targets. The ion temperature is assumed the same as the electron temperature at the targets, but it is calculated independently upstream. The flow velocity at the targets is assumed to be sound speed (Mach number $=1$ ) in accordance with the Bohm criterion. A recycling coefficient of 1 was assumed. 
The Langmuir probe data for the discharge \#22575 and the reference discharge \#22469 are shown in Figure 1. At the inner target, the Langmuir probe data are less reliable, due to the fact that the probes are at a very shallow angle to the magnetic field [7]. Therefore, all data which were clearly affected by the shallow angle were removed when determining the input. Another possible complication is that the inner divertor was partially detached, a condition not well modeled by OSM solver 22. Because of these reasons, two different plasma backgrounds were considered in this work. The first one is an asymmetric case where the values shown in Figure 1 are given to the OSM solver. The other background is a symmetric case where the more reliable experimental data from the outer target is used as input also for the inner target. Similar sensitivity tests were also carried out by multiplying the target density or temperature by a constant factor.

The generated plasma background for the asymmetric case was compared to experimental measurements at the outer midplane. Electron and ion temperature were reproduced but the electron density was too low by a factor of 2 in the near SOL and almost by a factor 10 in the far SOL compared to measurements. This discrepancy is likely to affect the simulation results. It should be noted, however, that various divertor conditions and, consequently, various upstream conditions were also simulated. The main point of this work was to identify key parameters for impurity transport.

The generated plasma background had a poloidal ion temperature profile that peaks at the top of the plasma at roughly $100 \mathrm{eV}$. The poloidal flow profile increased towards the divertors with a fairly stagnant plasma upstream above the X-point (Figure 2). Measurements in ASDEX Upgrade [8,9] and in other devices [10] have, however, indicated flow profiles with a stagnation point between the outer midplane and the $\mathrm{X}$-point and a flow velocity of roughly $M=0.5$ at the high field side and the top of the plasma. The flow profile can be assumed to play an important role in the deposition profile. Therefore, tests were carried out with an ad-hoc imposed flow field in a similar fashion to [11]. 


\section{Simulation and Experimental Results}

To start with, the assumed radial injection location, the diffusion coefficient, and the ion periphery option were scanned. These are hereafter called the simulation parameters. Figure $3 \mathrm{a}$ shows the deposition from four DIVIMP cases with different simulation parameters but using the same asymmetric divertor conditions.

The simulation parameters did have a noticeable effect on the deposition results, but it was observed that their combined effect can almost be described by one variable, $\tau_{\mathrm{SOL}}$, the SOL dwelling time. Noting that perpendicular transport is modeled by a diffusion model, one can estimate the time it takes for an ion to exit the grid to be of the order of

$$
\tau_{\mathrm{SOL}} \approx \frac{(\Delta R+\mathrm{ipo})^{2}}{D}
$$

where $\Delta R$ is the perpendicular distance to the grid edge from the injection location, ipo is the specified ion periphery option halo plasma width, and $D$ is the perpendicular diffusion coefficient. The four cases in figure $3 \mathrm{a}$ had different simulation parameters, but they were chosen so that A1 and A2 had the same $\tau_{\mathrm{SOL}}$, as did B1 and B2. Simulations with the same $\tau_{\mathrm{SOL}}$ showed a similar deposition pattern. The same qualitative behaviour of the deposition pattern on $\tau_{\mathrm{SOL}}$ was also observed using different Langmuir probe input (results not shown in figure). This dependence on $\tau_{\mathrm{SOL}}$ makes the simulation parameter scans more reliable.

Using a low $\tau_{\mathrm{SOL}}$, most of the deposition occurs next to the injection location - as could be expected. With a higher $\tau_{\mathrm{SOL}}$, the deposition is shifted towards the top of the machine, but virtually nothing is deposited on the divertors. The injected impurities are transported to the top of the machine by the ion temperature gradient force in the fairly stagnant plasma. No good match to the experimental data was found with any realistic simulation parameters when using the asymmetric, default plasma background.

The effect of changing the Langmuir probe input profiles at the targets was tested using constant simulation parameters. The injection location was $1.0 \mathrm{~cm}$ from the separatrix, the diffusion coefficient was $0.5 \mathrm{~m}^{2} \mathrm{~s}^{-1}$, and the ion periphery option halo plasma width was $1 \mathrm{~cm}$. Figure 3B shows the deposition results from the asymmetric and symmetric cases. Changing 
from the asymmetric to symmetric case shifted the deposition in the high field side towards the top of the machine. This is likely due to the ion temperature gradient force being stronger when using higher divertor temperatures as input. The large-scale deposition on various parts of the machine or the qualitative deposition profile did not change noticeably, however. Tests were also carried out where the density or temperature was multiplied by $0.5,2$, or 5 at both targets (not shown in figures). The resulting deposition did not change noticeably in these runs.

The lack of flows in present edge simulations is recognized as an important issue, and simulations were carried out with an ad-hoc imposed flow field as in Elder et al. [11]. Figure 3B shows the deposition from a case where a flow field was imposed on the asymmetric OSMgenerated plasma background. The assumed flow velocity of the plasma background had a constant value of 0.5 Mach towards the divertors and a stagnation point about midway between the lower X-point and the outer midplane. The deposition profile changed dramatically compared to simulation cases without an imposed flow field. More was deposited at the inner divertor, the heat shield, and (perhaps surprisingly) also at the outer divertor. The difference between the symmetric and asymmetric cases with an imposed flow field was, however, small (not shown in figure). A fairly good match to the experimental deposition data was obtained using the imposed flow field. The experimental deposition to various parts of the machine was obtained by integrating over the poloidal cross section and then toroidally over the torus [2]. Measurements were scarce in the upper parts of the machine and the deposition was linearly interpolated between the measurement points. Of the injected ${ }^{13} \mathrm{C}$ atoms, $0.6 \%$ was found at the heat shield, $0.8 \%$ at the inner lower divertor, $0.1 \%$ at the private flux regions, $0.1 \%$ at the outer lower divertor, $0.3 \%$ at the limiter area, and $0.04 \%$ at the PSL area. There was only one measurement location at the upper divertor and the deposition was estimated to be between 1 $\%$ and $6 \%$ of the injected atoms. In total, only 3 to $8 \%$ of the injected atoms were accounted for in this manner. Possible reasons for this large missing fraction include deposition already inside the injection port and deposition in between the tiles. According to the arguments given in section 1, a toroidally fairly symmetric deposition is expected, but toroidal asymmetry cannot completely be ruled out. In the simulations, $100 \%$ of the injected ions are deposited on 
the walls. In the experiment, on the other hand, the numbers presented above could indicate that the majority never reaches the SOL and are deposited already inside the injection port. Both the experimental and simulated deposition were therefore normalized to $100 \%$ in order to make a comparison feasible. Since the upper divertor data is uncertain, it was left out from the normalization. Assuming the simulations actually replicate the experiments, this normalization scheme should nonetheless produce matching results to experimental values. That is, the ratios between between the inner and lower divertor, or the heat shield and limiter, for example, should be correct. Comparing how much is deposited in the simulations in the upper divertor compared to other parts gives an estimate of whether the experimental deposition should be closer to 1 or $6 \%$.

Figure 4 shows the best match obtained. The simulation parameters were $R=0.4 \mathrm{~cm}$ away from the separatrix, $D=0.25 \mathrm{~m}^{2} \mathrm{~s}^{-1}$, and ipo $=1 \mathrm{~cm}$ corresponding to $\tau_{\mathrm{SOL}}=1.6 \mathrm{~ms}$. The plasma background was the asymmetric one with the imposed flow field. Simulations using the symmetric divertor conditions and the imposed flow field produced almost identical results. In the simulations that matched the experimental results, roughly $23 \%$ of the injected atoms were deposited at the upper divertor. Considering the normalization, this approximately correspond to a measurement of $0.5 \%$ of the injected atoms. This result weakly indicates the experimental deposition is closer to $1 \%$ compared to $6 \%$.

\section{Summary}

In this paper, we simulated the 2007 global ${ }^{13} \mathrm{C}$ injection experiment at ASDEX Upgrade with DIVIMP. The plasma background was obtained using DIVIMP's onion skin model 22. The injection location of the simulated ions, the perpendicular diffusion coefficient, the ion periphery option, and the Langmuir probe data input for the plasma solver were scanned. We were not able to replicate the experimental deposition data in this manner.

However, Imposing an ad-hoc flow velocity into the generated plasma background, as done in [11], produced a fairly good match to the experimental deposition data using realistic values for the simulation parameters. This result strongly identifies the need for a better understanding of parallel background plasma flows in the SOL. 


\section{Acknowledgements}

This work was partially funded by the Academy of Finland. This work was supported by EURATOM and carried out within the framework of the Task Force on Plasma Wall Interactions of the European Fusion Development Agreement (EFDA). The view and opinions expressed herein do not necessarily reflect those of the European Commission.

\section{References}

[1] A. Herrmann, O Gruber Fusion Science and Techonology 44 (2003) 569 - 577

[2] A. Hakola et al Plasma Phys. Control. Fusion 52 (2010) 065006

[3] P. Stangeby, C. Farrell, S. Hoskins, L. Wood, Nuclear Fusion 28 (1988) 1945

[4] P.C. Stangeby et al., J. Nucl. Mater. 313 - 316 (2003) 883 - 887

[5] http:// www.eirene.de (referenced 10.5.2010)

[6] http://starfire.utias.utoronto.ca/divimp/docs/divdocs.html (referenced 20.07.2010)

[7] Personal communications, Dr. H. W. Müller

[8] H. W. Müller et al J. Nucl. Mater. 363 - 365 (2007) 605 - 610

[9] M. Tsalas et al. Plasma Phys. Control. Fusion 49 (2007) 857

[10] B. Lipschultz et al. Nucl. Fusion 47 (2007) 1189 - 1205

[11] J. D. Elder et al J. of Nucl. Mater. 363-365 (2007) 140 - 145 
Figure 1. The Langmuir probe data from the reference discharge \#22469 (light grey) and the discharge \#22575 (dark grey). The black solid line is the input given to DIVIMP. $\Delta s$ is the distance from the strike point. PP indicates the private plasma region.

Figure 2. The plasma background flow profile from the inner to outer divertor along a magnetic field line. The asymmetric and symmetric cases along with experimental measurements from ASDEX Upgrade [8,9] (circles) and various tokamaks [10]. Radially, the flux tube was approximately $1.5 \mathrm{~mm}$ away from the separatrix at the outer midplane. There are radial variations in the flow profile, but the general qualitative pattern remains the same. Positive Mach numbers indicate flow towards the outer divertor.

Figure 3. Poloidal deposition profiles for various DIVIMP cases. The calculational grid does not extend all the way to the walls at every part of the machine, and the deposition is plotted as a function of where the particles exit the grid. a) Four DIVIMP cases with different simulation parameters using the same plasma background. Runs A1 and A2 had the same $\tau_{\mathrm{SOL}}$, as did runs B1 and B2. b) Three DIVIMP cases with the same simulation parameters but using different plasma backgrounds. The letters AS refer to the default, antisymmetric divertor conditions, while the letter $\mathrm{S}$ to the symmetric divertor conditions. The letters IF refer to imposed flow. For testing purposes, a flow field was imposed upon the generated plasma background. The thick black dashed line is at the outer midplane (injection location), the thick black solid line is at the top of the tokamak, the two thin lines are the strike point locations.

Figure 4. The normalized experimental and simulated deposition are shown by the black and white bars, respectively. The grey bar is the unnormalized simulated deposition for the upper divertor. This was the best match obtained during the simulations. The plasma background was the one with asymmetric target conditions and an imposed flow field. ID = inner divertor, $\mathrm{PP}=$ private plasma region, $\mathrm{OD}=$ outer divertor, $\mathrm{Lim}=$ limiter, $\mathrm{PSL}=$ passive stabilizer loop, UD = upper divertor, HS = heat shield. 


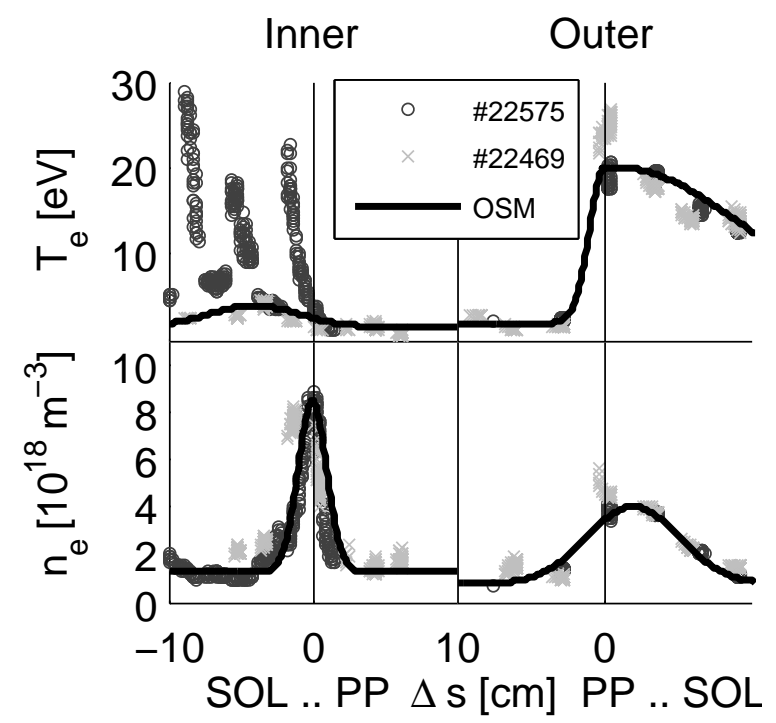

Figure 1: 


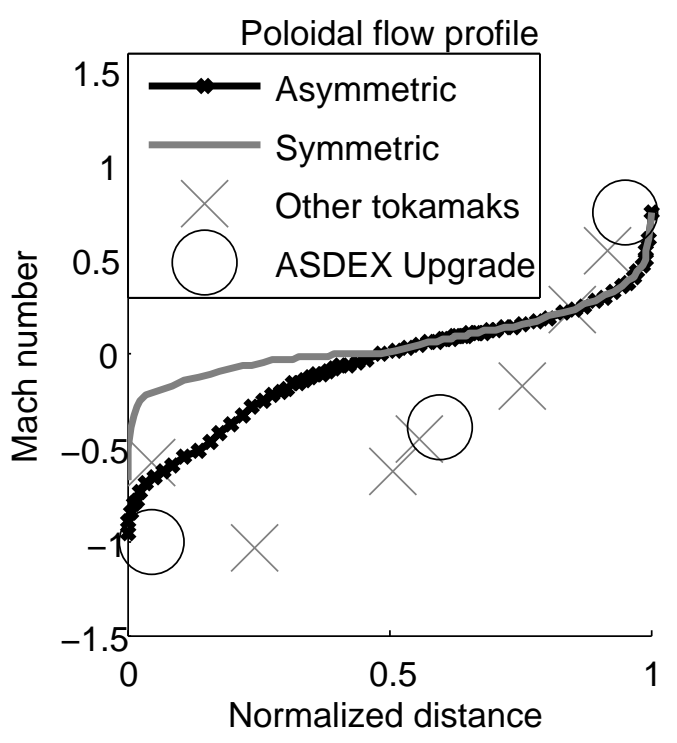

Figure 2: 


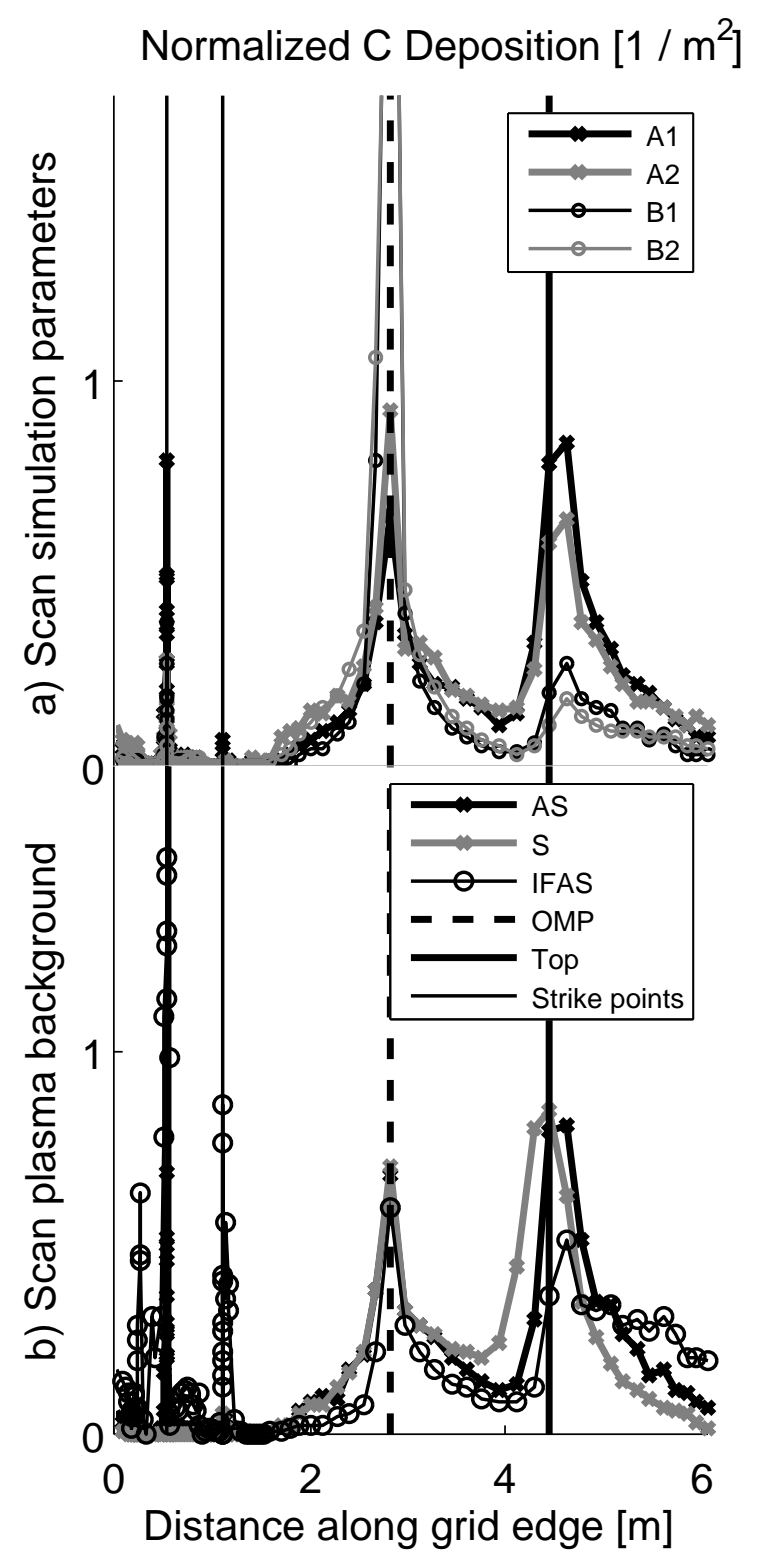

Figure 3: 


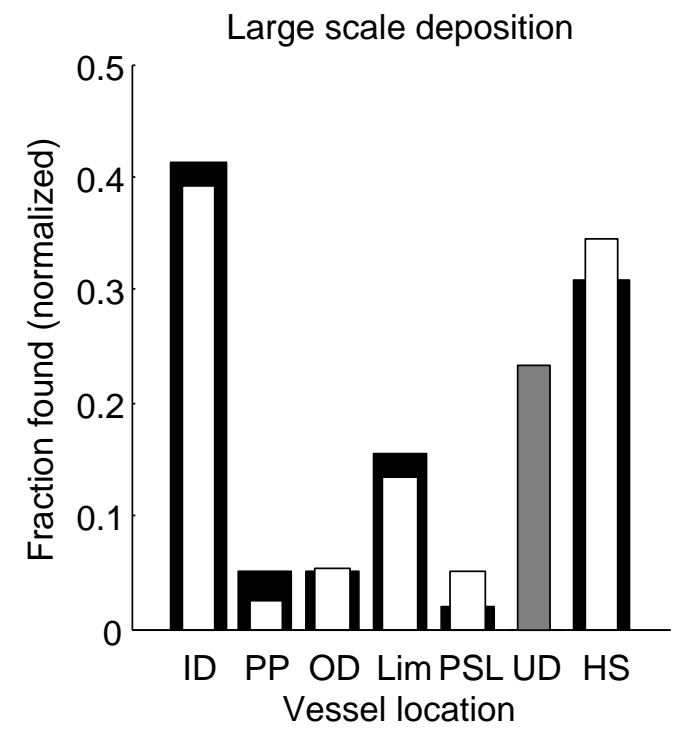

Figure 4: 